\title{
The Pathophysiology of Portal Hypertension
}

\author{
Gerhard Treiber Antal Csepregi Peter Malfertheiner \\ Department of Gastroenterology, Hepatology, and Infectious Diseases, University Hospital Magdeburg, \\ Magdeburg, Germany
}

\section{Key Words}

Portal hypertension · Vasoconstrictors · Vasodilators end up in the formation of collateral vessels. Varices can involve the whole gastrointestinal tract and are a fre-

quent source of bleeding.

\begin{abstract}
Portal hypertension is defined by an elevation in blood pressure in the portal system. Different causes are known and include a pre-, intra-, or posthepatic block. Portal hypertension is also classified according to the sinusoidal system. Portal pressure becomes elevated by either an increase in blood flow (Q), an increase in resistance $(R)$, or both. Regulation of the vascular tone in the splanchnic system includes intrinsic and extrinsic aspects. A variety of metabolic end-products (e.g. adenosine), endothelium-derived substances (e.g. nitric oxide), and certain neurotransmitters (e.g. acetylcholine) are known to relax the tone and thus produce vasodilation. Important vasoconstrictor influences on splanchnic arterioles include circulating agents (e.g. angiotensin), endothelium-derived substances (e.g. endothelin), and again neurotransmitters (norepinephrine). Besides vascular tone, structural changes (thrombosis, fibrosis, shear stress, and cell regeneration) add to overall hepatic resistance. Further consequences of portal hypertension include an increase in blood flow which leads to a hyperdynamic state with fluid retention, leading to secondary involvement of other organs, such as cirrhotic cardiomyopathy, hepatopulmonary syndrome and hepatorenal syndrome. Finally, portal hypertension will
\end{abstract}

Copyright (C) 2005 S. Karger AG, Basel

\section{Anatomical Aspects}

The portal venous system collects blood from the spleen and all organs participating in the digestion of food, such as the stomach, pancreas, small bowel, and the colon. Downstream from the anatomical region where all these veins meet (confluens region), the portal vein delivers substrate-rich venous blood towards the liver, where substrates undergo extensive metabolization. In the liver, the partially deoxygenated blood carried by the low pressure portal vein joins the well-oxygenated blood derived from the high pressure hepatic artery. Finally, hepatic veins will then drain the blood out of the liver. The organs perfused by the splanchnic vasculature receive about $25 \%$ of cardiac output (at resting conditions).

\section{Definition and Classification of Portal Hypertension}

Portal hypertension can be characterized in several ways. From an anatomical point of view, a block within the system could be localized at the presinusoidal, sinu-

\section{KARGER \\ Fax +4161306 1234 E-Mail karger@karger.ch} www.karger.com
Gerhard Treiber, MD, Department of Gastroenterology,

Hepatology, and Infectious Diseases, University Hospital Magdeburg

Leipziger Strasse 44, DE-39120 Magdeburg (Germany)

Tel. +49 3916713100, Fax +49 3916713105

E-Mail gerhard.treiber@medizin.uni-magdeburg.de 
Table 1. Classification of portal hypertension

a Classification of $\mathrm{PH}$ based on anatomical considerations

\section{Prehepatic causes}

Portal vein thrombosis

Splanchnic arteriovenous fistula

Splenic vein thrombosis

Splenomegaly due to other systemic diseases

Intrahepatic causes

Chronic hepatitis irrespective of cause

Budd-Chiari syndrome

Drug toxicity

Fulminant liver failure

Hepatic veno-occlusive disease

Malignant or metastatic hepatic diseases

Myeloproliferative diseases

Nodular regenerative hyperplasia

Peliosis hepatis

Primary biliary cirrhosis

Sarcoidosis

Schistosomiasis

Wilson's disease

Posthepatic

Cardiac disease

(constrictive pericarditis, restrictive cardiomyopathy)

Inferior vena cava obstruction

b Classification of portal hypertension based on functional considerations

Presinusoidal

Extrahepatic portal vein obstruction

Pancreatitis, tumor, biliary tract disease

Phlebitis, omphalitis

Hypercoagulable state

Associated cirrhosis

Segmental portal hypertension (such as splenic vein thrombosis)

Intrahepatic portal vein obstruction

Schistosomiasis

Noncirrhotic portal fibrosis

Primary biliary cirrhosis

Sarcoidosis

Sclerosing cholangitis

Congenital hepatic fibrosis

Hepatic arterioportal fistula

Noncirrhotic sinusoidal portal hypertension

Acute hepatitis

Vitamin A toxicity

Arsenic poisoning

Vinyl chloride toxicity

Secondary syphilis

Nodular regenerative hyperplasia

Postsinusoidal portal hypertension

Veno-occlusive disease

Budd-Chiari syndrome

Constructive pericarditis

Restrictive cardiomyopathy

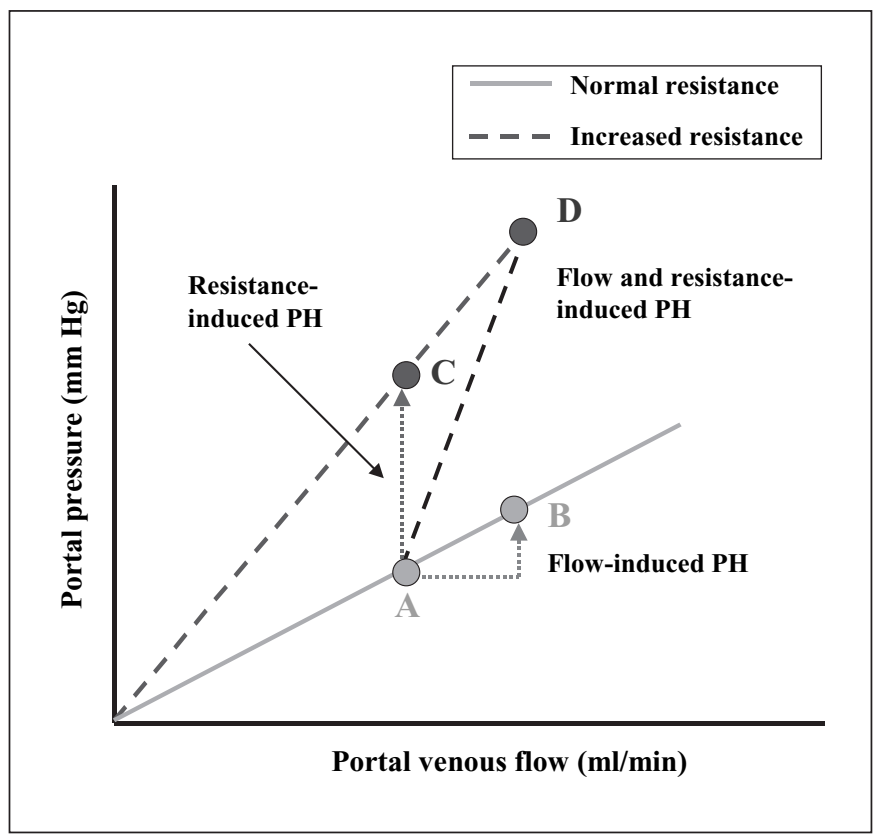

Fig. 1. Hepatic resistance and portal venous flow determine portal pressure (pP). Circle A represents normal portal venous flow with normal portal pressure, the increase of $\mathrm{pP}$ is only induced by an increase in flow $(\mathrm{B})$ or resistance $(\mathrm{C})$; circle $\mathrm{D}$, in contrast, represents an elevation in both. $\mathrm{PH}=$ Portal hypertension. Modified from references $1-7$.

soidal, or postsinusoidal level. Major causes are depicted in table 1. If the block arises outside the liver or portal vein, the term 'partial' or 'isolated' portal hypertension is used; it is mainly caused by thrombosis of the splenic vein, e.g. following necrosing pancreatitis. Although patients with this condition can develop gastric or esophageal varices as well, they usually do not show other characteristic signs of portal hypertension.

To estimate the severity of portal hypertension, invasive measurements by the wedged hepatic venous pressure can be performed. An elevation $\geq 12 \mathrm{~mm} \mathrm{Hg}$ is associated with an increased risk of variceal bleeding; however, in the presence of a prehepatic or presinusoidal block, these measurements fail to indicate portal hypertension. Here, direct invasive or noninvasive measurements of variceal pressure are preferred.

From a mechanistic view, portal pressure $(\mathrm{pP})$ may become elevated by increases in blood flow (Q) and/or increase in resistance ( $\mathrm{R}): \Delta \mathrm{pP}=\mathrm{Q}$ (accounting for about $40 \%) \times \mathrm{R}$ (accounting for about 60\%).Typical changes are shown in figure 1 .

The regulation of the splanchnic arterioles, which are partially constricted under normal conditions, underlies 
Fig. 2. NO-derived regulation of vascular tone in endothelial smooth muscle cells. Modified from references 1-7.

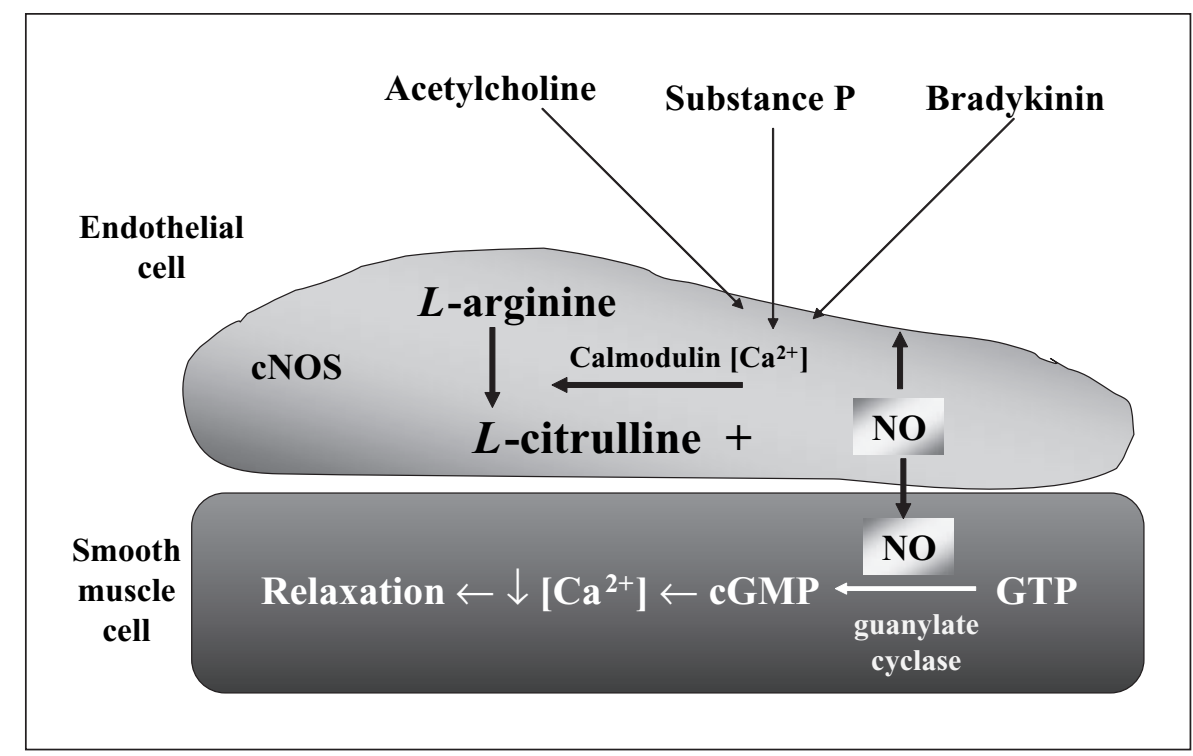

multiple factors known to influence the contractile state (tone) of the arteriolar smooth muscle. Here nitric oxide (NO) plays a central role (fig. 2).

In the splanchnic system, arterioles are about 10 times more potent in regulating overall pressure than venules (in contrast to other body compartments where a 1:1 ratio is observed). A variety of metabolic end-products (e.g. adenosine), some endothelium-derived substances (e.g. NO), and certain neurotransmitters (e.g. acetylcholine) are known to relax the tone and thus produce vasodilation. Important vasoconstrictor influences on splanchnic arterioles include circulating agents (e.g. angiotensin), endothelium-derived substances (e.g. endothelin), and again neurotransmitters (norepinephrine). An overview is given by table 2. If smooth muscle tension is the controlled variable, intravascular pressure can be understood as a determinant of arteriolar diameter (myogenic regulation), where tension $(\mathrm{T})$ is $=$ intravascular pressure $\mathrm{P} \times$ vessel radius $r$.

Hence, a sudden elevation of portal pressure in an otherwise normal individual elicits the constriction of arterioles in those organs draining blood into the portal vein. However, this holds mainly true for normal conditions and describes acute changes mediated by hypoxia and increased metabolites such as adenosine. In chronic liver disease, the vascular response contrasts with the intense dilation of arterioles that accompanies chronic portal hypertension. Here, the accumulation of vasodilator influences (e.g. NO, glucagon) dominate over myogenic vasoconstrictor influences that are intrinsic to the splanchnic
Table 2. Factors influencing the development and severity of portal hypertension

Circulating vasoconstrictors
Norepinephrine
Angiotensin II
Vasopressin (ADH)
Endothelin 1
Circulating vasodilators
Glucagon
Prostaglandins
VIP
Substance P
Metabolic mediators
Hypoxia
Adenosine
Acidosis
Cysteinyl leukotrienes
Thromboxane $\mathrm{A}_{2}$
Endothelium-derived factors
Endothelin 1 via ADH and thrombin
NO via L-arginine regulated by acetylcholine,
substance P and bradykinin

Norepinephrine

Angiotensin II

Vasopressin (ADH)

Glucagon

Prostaglandins

VIP

Metabolic mediators

Hypoxia

Adenosine

Cysteinyl leukotrienes

Endothelium-derived factors

Endothelin 1 via ADH and thrombin substance $P$ and bradykinin circulation. As a result, the splanchnic arteriolar diameter is increased, vascular resistance is low and blood flow is increased (increased endothelial 'eNOS'-derived NO synthesis, decreasing vascular tone). These changes are accompanied by changes in the hepatic microvasculature. With continuing inflammation and development of fibro- 
Fig. 3. Overview of mechanisms contributing to portal hypertension. Modified from references $1-7$.

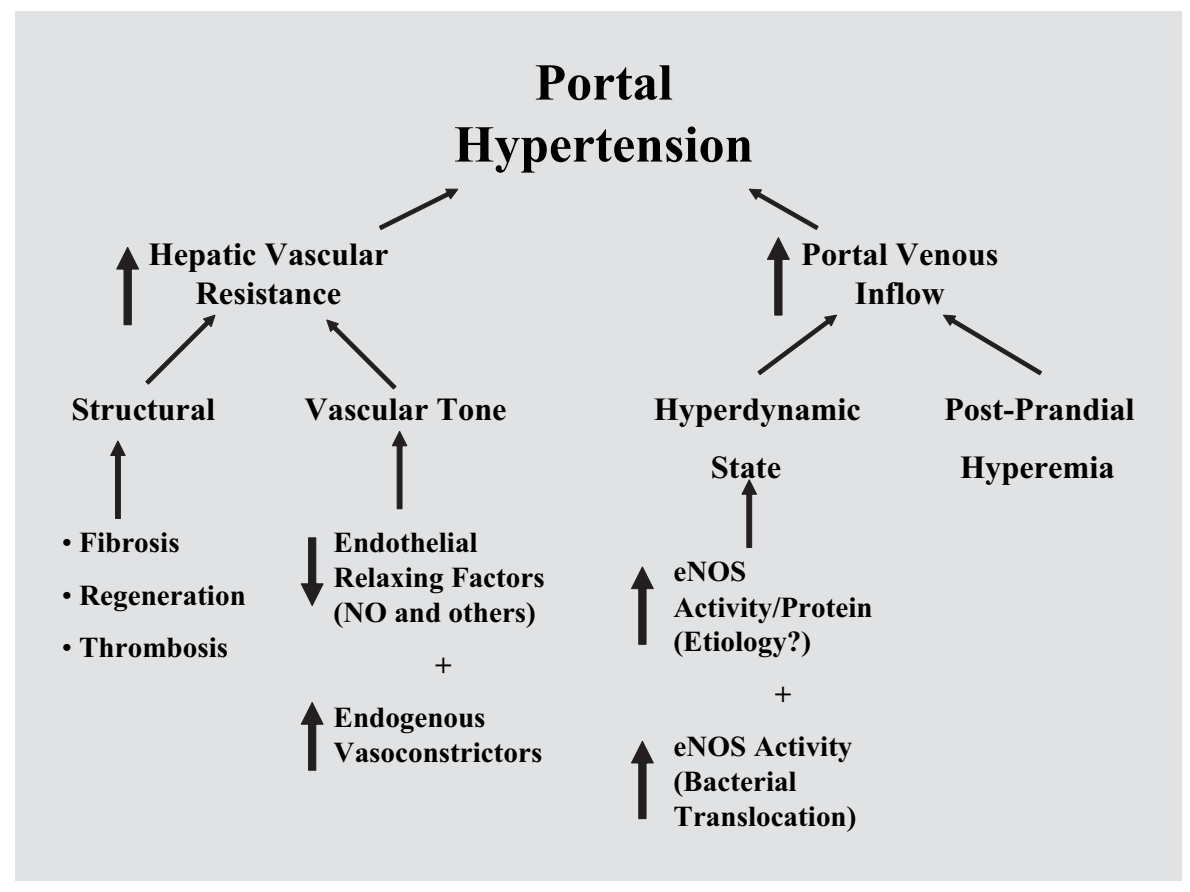

sis, the hepatic parenchyma diminishes and the functional status of the remaining liver cells is additionally impaired by shear stress. This leads to reduced eNOSderived NO synthesis in the liver bed and the microcirculatory NO deficiency leads to an increase in vascular tone, amplifying the structural changes described. Therefore the balance between vascular tone in the liver and the splanchnic system, although shifting to the opposite direction, will eventually have the same clinical consequence, i.e. the development of portal hypertension.

The extrinsic regulation of splanchnic blood flow is characterized by the fact that circulating vasoconstrictors are the major contributors to basal splanchnic arteriolar tone: about $60 \%$ of total impact is derived from norepinephrine, angiotensin II, and vasopressin. In contrast, in the brain, these factors only account for $5 \%$ of total regulation. Next, regulation mediated by circulating vasodilators (glucagon, VIP, substance P) contributes substantially to splanchnic hyperemia in some forms, because impaired liver function will decrease hepatic degradation (e.g. for glucagon). Together with the increased production of reactive autonomous vasodilators such as $\mathrm{PGI}_{2}$, this explains why the splanchnic vasculature will become hyporeactive to vasoconstrictors such as norepinephrine, vasopressin, angiotensin II, and endothelin-1. In summary, these factors will increase portal blood flow. In comparison, as food ingestion will release the same variety of GI hormones and peptides to produce rapid transient arteriole dilation, liver cirrhosis and other causes of PH will lead to permanent elevation of these factors. The clinical observation that variceal rupture and bleeding often occur after meals can be attributed to the fact that all compensatory mechanisms are already at maximum use and any further increase in blood flow will then have a deleterious clinical impact.

However, splanchnic vasodilation has further consequences: significant venous blood pooling develops and is followed by systemic vasodilation and arterial underfilling. Together with the indirect cause of secondary hyperaldosteronism induced by impaired hepatic degradation, the lack of intravascular volume will activate the renin-angiotensin-aldosterone system, leading to sodium and water retention. This increase in total blood volume will lead to a hyperdynamic state, again increasing portal venous inflow. These conclusions are supported by animal experiments showing that NOS activity is increased in cirrhosis and that the reversal of primary arterial vasodilation in experimental cirrhosis is possible with NOS inhibition. Furthermore, NOS inhibition in experimental cirrhosis reverses the hyperdynamic circulation, the activation of the neurohumoral axis and sodium and water retention. 


\section{Summary (fig. 3)}

Portal hypertension is caused by either an increase in hepatic vascular resistance or portal venous inflow. Therefore, structural changes (fibrosis, thrombosis) together with increased vascular tone mediated by intrinsic and extrinsic vasoconstrictors and vasodilators are accompanied by a hyperdynamic state due to arterial underfilling and secondary fluid retention. Further consequences of portal hypertension include an increase in blood flow which will lead to a hyperdynamic state with fluid retention, leading to secondary involvement of other organs, such as cirrhotic cardiomyopathy, hepatopulmonary syndrome and hepatorenal syndrome. Finally, portal hypertension will end up in the formation of collateral vessels (varices). The described mechanism(s) provide exciting perspectives for therapeutic interventions that are already established (e.g. $\beta$-blockers) or emerging (e.g. endothelin receptor antagonists).

\section{References}

-1 El Atti EA, Nevens F, Bogaerts K, Verbeke G, Fevery $\mathrm{J}$ : Variceal pressure is a strong predictor of variceal haemorrhage in patients with cirrhosis as well as in patients with non-cirrhotic portal hypertension. Gut 1999;45:618-621.

-2 Nevens F, Bustami R, Scheys I, Lesaffre E, Fevery $\mathrm{J}$ : Variceal pressure is a factor predicting the risk of a first variceal bleeding: A prospective cohort study in cirrhotic patients. Hepatology 1998;27:15-19.
-3 Wiest R, Groszmann RJ: The paradox of nitric oxide in cirrhosis and portal hypertension: too much, not enough. Hepatology 2002;35:478491.

-4 Moreau R, Lebrec D: Endogenous factors involved in the control of arterial tone in cirrhosis. J Hepatol 1995;22:370-376.

5 Schrier RW, Gurevich AK, Cadnapaphornchai MA: Pathogenesis and management of sodium and water retention in cardiac failure and cirrhosis. Semin Nephrol 2001;21:157-172.
-6 Mesh CL, Joh T, Korthuis RJ, Granger DN, Benoit JN: Intestinal vascular sensitivity to vasopressin in portal hypertensive rats. Gastroenterology 1991;100:916-921.

-7 Atucha NM, Shah V, Garcia-Cardena G, Sessa WE, Groszmann RJ: Role of endothelium in the abnormal response of mesenteric vessels in rats with portal hypertension and liver cirrhosis. Gastroenterology 1996;111:16271632 . 